\title{
Lack of Improvement: A National Assessment of Readmission Rates After Pediatric Bladder Reconstruction
}

\author{
Seth Cohen ${ }^{1}$; Kerrin Palazzi ${ }^{2}$; Sarah Marietti ${ }^{1,2}$; George Kaplan ${ }^{1,2}$; George Chiang ${ }^{1,2, *}$ \\ ${ }^{1}$ Department of Urology, UC San Diego Health System, University of California, San Diego, USA \\ 2 Pediatric Urology, Rady Children's Hospital-San Diego, University of California, San Diego, USA \\ ${ }^{*}$ Corresponding author: George Chiang, Department of Urology, UC San Diego Health System, University of California, San Diego, USA. Tel: +1-8589665922, Fax: +1-8589668479, E-mail: \\ gchiang@rchsd.org
}

Received: May 16, 2014; Accepted: June 14, 2014

\begin{abstract}
Background: Bladder reconstruction in the pediatric population is challenging for many reasons, including perioperative complications and readmissions.

Objectives: On a national scale, determine readmission rates at 30,60 and 90 days after bladder reconstruction in a pediatric population over a 7-year period, evaluating the influence of hospital and patient-specific variables.

Patients and Methods: Using the Pediatric Health Information System database, we identified patients 0-17 years of age, from 20042010, undergoing bladder reconstruction using ICD-9 procedure codes. Descriptive statistics characterized demographics, prevalence of surgeries, and readmission rates. Surgery prevalence over time was examined using linear regression. Readmission rates were compared using the Chi2 test. Regression was used to evaluate the influence of variables on readmission risk.

Results: We identified 1,985 patients for inclusion, of which 52.7\% were female. Median age was 9 years. There has been no change in the prevalence of bladder reconstruction surgeries $(\mathrm{P}=0.327)$. There was no change in 30-day $(\mathrm{P}=0.272), 60$-day $(\mathrm{P}=0.788)$ or 90-day readmission rates $(\mathrm{P}=0.924)$. Despite surgical volume adjustment, 90-day readmission rates did not significantly vary among the majority of hospitals. Initial LOS $>7$ days $(\mathrm{P}<0.001)$ and complex chronic condition males $(\mathrm{P}<0.001)$ were significantly associated with 90-day readmission.

Conclusions: No improvement in readmission rates after pediatric bladder reconstruction was observed during the study period. Nearly all centers have a similar readmission rate despite volume adjustment.
\end{abstract}

Keywords:Urology; Bladder; Complications; Database

\section{Background}

While augmentation cystoplasty has seen its indications decrease with the advent of onabotulinum toxin A and neuromodulation, there remains a role for this procedure in the 21st century (1). Analysis from the Nationwide Inpatient Sample (NIS) reveals that augmentation cystoplasty appears to be widely used in the surgical management of neurogenic bladder in patients with spina bifida (2). Data from the Pediatric Health Information System (PHIS) database demonstrates no change in augmentation rates between 1999 and 2004, finding 665 patients who underwent augmentation cystoplasty over this 5-year period (3). A recent study by UC San Francisco demonstrated a decrease in the number of augmentations by $25 \%$ in the 2000 s (4). Institutional case series have examined complications of bladder reconstruction, suggesting it continues to have notable morbidity. Obermayr et al. retrospectively analyzed 25 pediatric patients who underwent augmentation cystoplasty (5) and bladder substitution (6) between 1999 and 2007, finding major complications including lower urinary tract calculi (39\%), stricture or insufficiency of the continent vesicostomy (28\%), and intestinal obstruction (9\%) (5). Approximately one-third of patients who have undergone en- terocystoplasty experience postoperative complications $(6,7)$. These morbidities are secondary to a multitude of factors including surgical technique, inherent properties of the bowel segment, and patient compliance with maintenance regimens (8).

\section{Objectives}

We sought to determine, on a national scale, the readmission rates at 30, 60 and 90 days after initial surgery, whether they have improved over the 7-year period, and to determine the influence of hospital and patient-specific variables with estimated costs of readmission.

\section{Patients and Methods}

\subsection{Pediatric Health Information System}

This was a retrospective cohort analysis of the PHIS, a national administrative database containing combined hospitalization data from 43 tertiary care pediatric hospitals in the United States. This study was reviewed and approved by the UC San Diego Human Research Protections Program Institutional Review Board (IRB) with an informed consent waiver. Patients were followed across multiple hospital admissions to the same PHIS hospital

Copyright (C) 2014, Nephrology and Urology Research Center; Published by Kowsar Corp. This is an open-access article distributed under the terms of the Creative Commons Attribution License, which permits unrestricted use, distribution, and reproduction in any medium, provided the original work is properly cited. 
using unique numerical patient identifiers. Patient privacy and confidentiality are protected and this study conforms to the ethical guidelines of the 1975 Declaration of Helsinki. Inpatient data, emergency room visits, observation, and ambulatory surgery/medicine visits are all included. Some hospitals contribute varying amounts of data, for example, only inpatient visits versus inpatient, ambulatory, emergency room, and observation visits. Each participating institution and an independent data management group review the data for accuracy each quarter. Data is only accepted into PHIS if classified errors occur in less than $2 \%$ of the quarterly data. Thirty-seven of the 43 hospitals had complete inpatient information during the study period and were included in the analysis.

\subsection{Data Collection and Analysis}

We identified patients 0-17 years of age, from 20042010, undergoing bladder reconstruction using ICD-9 procedure codes: 56.51 (formation cutaneous ureteroileostomy), 57.87 (reconstruction of urinary bladder), and 57.88 (other anastomosis of bladder); patients undergoing repair of bladder exstrophy (ICD9 57.86) were excluded. Descriptive statistics were used to characterize patient demographics, prevalence of surgeries, and readmission rates. Surgery prevalence was examined using linear regression and readmission rates were compared using the chi $^{2}$ test. Total charges were compared between those who required readmission and those who did not, using the Mann-Whitney u-test. Costs are recorded in the PHIS database using a cost-to-charges ratio (RCC-based) for each hospital, which standardizes costs nationally taking into account variability in pricing and costs regionally. Although the RCC method is a good approximation for true costs, these costs are estimates and as such are only used in this analysis to compare between groups, rather than reporting them as a true exact cost amount. Ninety-day readmission rates were compared between hospitals after adjusting for hospital volume of augmentation surgery (number of readmits/total number of augment surgeries). GEE (generalized estimating equation) regression was used to evaluate the influence of independent variables on 90-day readmission risk while adjusting for patient clustering within hospitals. Covariates entered into the original model included age, gender, race, insurance (public/ private), complex chronic condition (which comprises a broad array of ICD 9 codes for congenital anomalies) (9), length of stay ( $<=7,>7$ days), year of surgery, days admitted prior to surgery to accommodate for bowel preps ( 0 or 1) and all 2-term interactions. Only variables that remained significant in multivariate analysis remained in the final models. Significance for testing was set a priori at $\mathrm{P}<0.05$, and STATA ver. 11.2 (StatCorp LP, TX) was used for statistical analyses.

\section{Results}

We identified 1,985 patients who underwent bladder reconstruction between 2004 and 2010. Demographic and hospital characteristics are shown in Table 1 . Median age of the cohort was 9 years and $52.7 \%$ were female. Median length of stay was 7 days.

\begin{tabular}{|c|c|}
\hline & $\begin{array}{l}\text { Augment Surgery, } \\
\qquad(\mathrm{n}=\mathbf{1 , 9 8 5 )}\end{array}$ \\
\hline Median Age (IQR), y & $9(5-12)$ \\
\hline \multicolumn{2}{|l|}{ Gender } \\
\hline Male & $938(47.3)$ \\
\hline Female & $1047(52.7)$ \\
\hline \multicolumn{2}{|l|}{ Race } \\
\hline Caucasian & $1315(66.2)$ \\
\hline African-American & $190(9.6)$ \\
\hline Other & $479(24.1)$ \\
\hline Spina Bifida & $515(25.9)$ \\
\hline Complex Chronic Condition & $1,657(83.5)$ \\
\hline \multicolumn{2}{|l|}{ Insurance } \\
\hline Private & $744(37.5)$ \\
\hline Medicare & $50(2.5)$ \\
\hline Medicaid & $800(40.3)$ \\
\hline Other & $365(18.4)$ \\
\hline Academic Hospital & $1,960(98.7)$ \\
\hline \multicolumn{2}{|l|}{ Region } \\
\hline North Central & $897(45.2)$ \\
\hline NE & $274(13.8)$ \\
\hline South & $472(23.8)$ \\
\hline West & $342(17.2)$ \\
\hline \multicolumn{2}{|l|}{ Bed Size } \\
\hline Small $(<200)$ & $501(25.2)$ \\
\hline Medium (200-300) & $1,134(57.1)$ \\
\hline Large $(>300)$ & $350(17.6)$ \\
\hline \multicolumn{2}{|l|}{ Urinary Code } \\
\hline $\begin{array}{l}\text { 56.51 Formation of Uretero- } \\
\text { ileostomy }\end{array}$ & $107(5.4)$ \\
\hline $\begin{array}{l}\text { 57.87 Reconstruction of Urinary } \\
\text { Bladder }\end{array}$ & $1,379(69.5)$ \\
\hline $\begin{array}{l}57.88 \text { Other Anastomosis of } \\
\text { Bladder }\end{array}$ & $867(43.7)$ \\
\hline Multiple Urinary Codes & $365(18.4)$ \\
\hline Multiple Admissions for Surgery & $124(6.2)$ \\
\hline
\end{tabular}

$\mathrm{a}$ Data are presented as No. (\%).

Over 7 years, there has been no change in the prevalence of bladder reconstruction surgeries ( $\mathrm{R}^{2}$ : 0.191, $\beta$ coefficient:-4.71, 95\% CI:-15.9 to 6.4, $\mathrm{P}=0.327$; Figure 1). There was 
Cohen S et al.

no change in 30 -day (ranging from $14 \%$ to $20 \% \mathrm{P}=0.272$ ), 60 -day (19\% to $24 \%, \mathrm{P}=0.788$ ) or 90 -day readmission rates over time (23\% to $27 \%, \mathrm{P}=0.924$ ) (Table 2 ). Despite surgical volume adjustment, the majority of hospitals had similar 90-day readmission rates (Figure 2). From a total of 37 hospitals, two had significantly higher readmission rates/volume and three had significantly lower readmission rates/volume than the mean. The median cost for those patients requiring readmission was 1.76 -fold higher than that of patients who were not readmitted based on RCC $(\mathrm{P}<0.001)$.

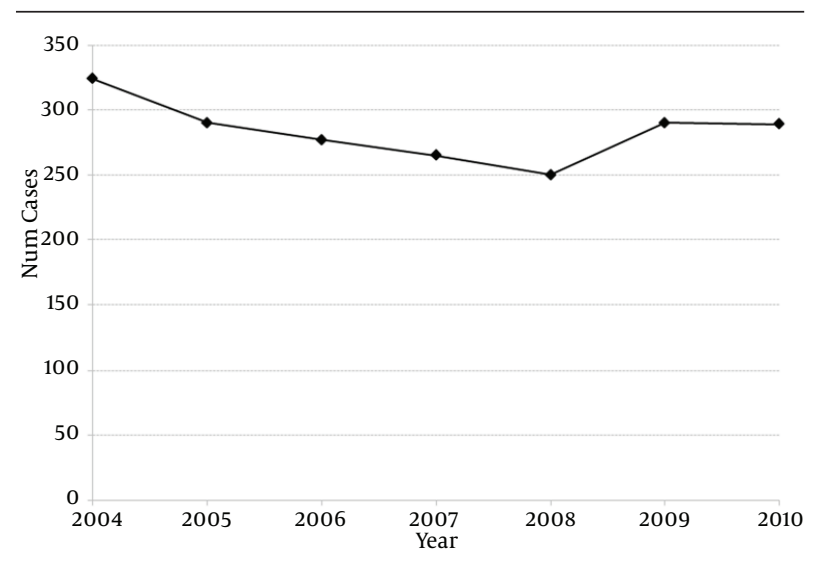

Figure 1. Bladder Augmentation Prevalence From 2004-2010
Table 2. 30-, 60- and 90-Day Readmission Rates (Unadjusted for Hospital Volume) ${ }^{\mathrm{a}}$

\begin{tabular}{lccc}
\hline & 30 Day & 60 Day & 90 Day \\
\hline $\mathbf{2 0 0 4}$ & $54(16.7)$ & $73(22.5)$ & $78(24.1)$ \\
$\mathbf{2 0 0 5}$ & $52(17.9)$ & $65(22.4)$ & $78(26.9)$ \\
$\mathbf{2 0 0 6}$ & $45(16.2)$ & $56(20.2)$ & $67(24.2)$ \\
$\mathbf{2 0 0 7}$ & $53(20)$ & $58(21.9)$ & $70(26.4)$ \\
$\mathbf{2 0 0 8}$ & $34(13.6)$ & $47(18.8)$ & $60(24)$ \\
$\mathbf{2 0 0 9}$ & $59(20.3)$ & $69(23.8)$ & $76(26.2)$ \\
$\mathbf{2 0 1 0}$ & $42(14.5)$ & $57(19.7)$ & $67(23.2)$ \\
\hline P Value & 0.272 & 0.788 & 0.924 \\
\hline Overall & 17.1 & 21.4 & 25 \\
\hline
\end{tabular}

${ }^{a}$ Data are presented as No. (\%) or \%.

On multivariate analysis, a significant interaction was seen between gender and complex chronic condition (CCC) and as such, additional models were generated after splitting by gender: initial LOS $>7$ days had 2 -fold higher odds of readmission than $<=7$ (males OR 1.647, P $=0.001$; females OR 2.18, $\mathrm{P}<0.001)$. Within males, having a CCC was significantly associated with 90-day readmission (OR 1.898, P = 0.001); CCC was not an independent predictor in females $(\mathrm{P}=0.147)$.

Figure 2. Volume Adjusted Rates of 90-day Readmission Per Hospital

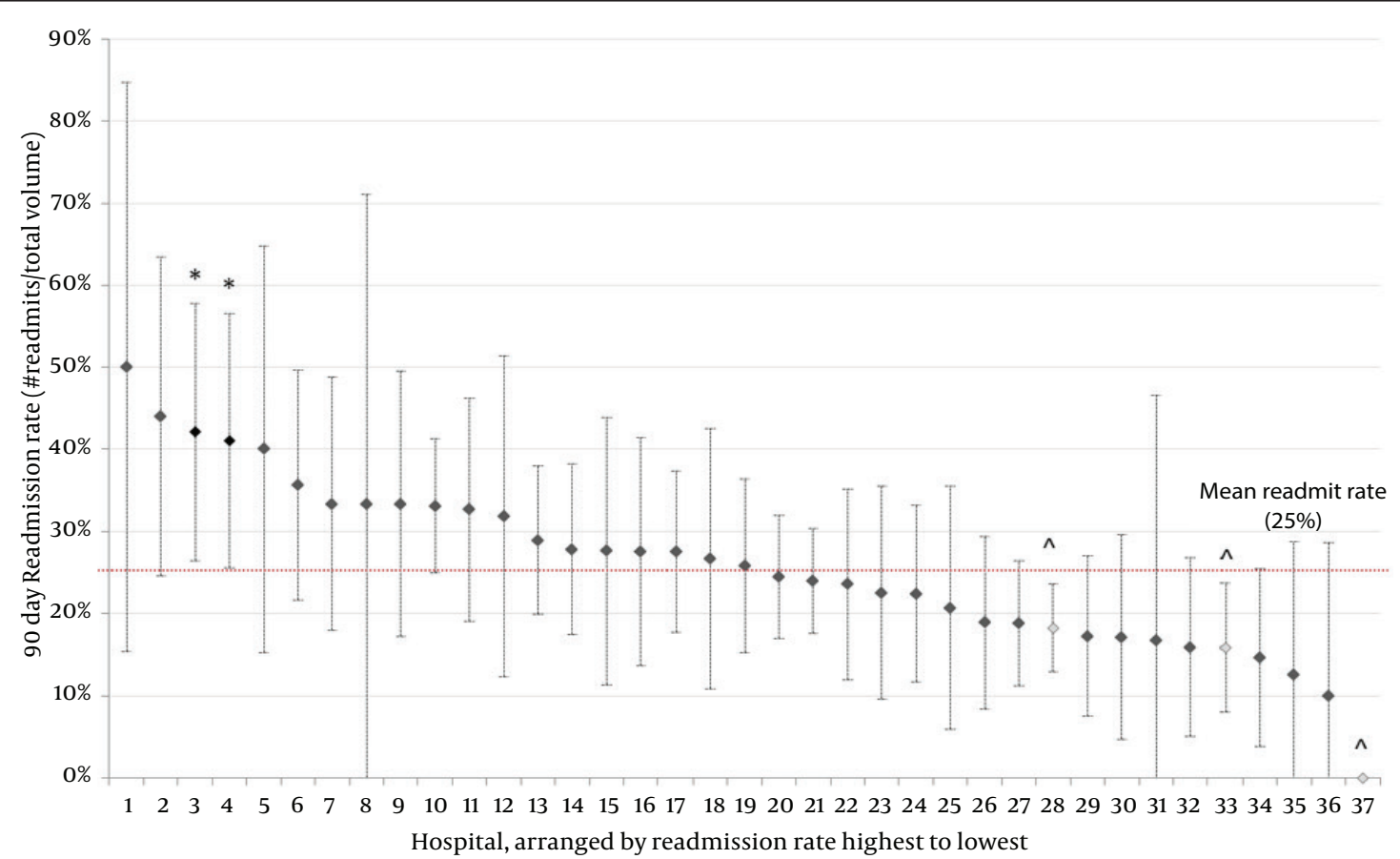

2 hospitals had significantly higher readmission rate than the mean $(*)$, and 3 had significantly lower readmission rates $\left({ }^{\wedge}\right)$. 


\section{Discussion}

Between 2004 and 2010, there was no significant change in the prevalence in bladder reconstruction in the pediatric population, nor was there any change in the rate of readmission of these post-surgical patients at 30,60 , or 90 days. A recent study by Schlomer et al. shows a $25 \%$ decrease in bladder augmentation over the last decade (4). This may be accounted for by the different cohorts analyzed. However, the critical issue of this study is the need for early postoperative readmission, which has been stable and alarmingly high. Beyond the direct morbidity to the patient, there is also a tremendous cost to the health care system with a 1.7-fold increase over patients who are not readmitted. The 90-day admission rate of $23-27 \%$ is comparable to the $30 \%$ readmission rate seen following either adult kidney or liver transplantation, which speaks to the severity of the condition and surgery $(10,11)$. Following urinary diversion in adults, the 90-day readmission rate has also been reported to be near 30\% as well (12). When counseling families and practitioners, the significant likelihood of readmission should not be underestimated.

\subsection{Readmission Rates}

The reasons for early readmission are not clearly elucidated in this study. Single institutional studies have been able to identify long-term morbidity following reconstruction. In adults, Welk et al. reported the rate of additional urological surgery after adult enterocystoplasty, finding $40 \%$ of patients required subsequent urological surgery in 243 patients, with a median follow up of 7.8 years (13). Similar to adults, there is impressive morbidity after total bladder substitution in the pediatric population. DeFoor et al. retrospectively analyzed a population of 26 patients, median age 8.9 years, with median follow up of 8 years, who underwent neobladder creation, finding complications including reservoir stones (8 patients), febrile urinary tract infections (8 patients), metabolic acidosis (6 patients), small bowel obstruction (4 patients), bladder perforation (4 patients), and pelvic lymphocele (1 patient) (14). Although the short- to long-term complications following pediatric bladder augmentation/ reconstruction have been enumerated, the likelihood of early readmission following initial surgery has not been studied from a population-based perspective. Despite including 37 PHIS hospitals nationwide in our analysis with a diverse volume of bladder augmentation caseloads, our analysis found no appreciable difference in postoperative readmission rates among hospitals. This could be secondary to several reasons, including the consistency of pediatric urologists at the majority of PHIS hospitals, the essential high morbidity of the surgery itself and its predisposing condition, or even the likelihood that an increased presence of patients with CCCs at high volume centers may dilute the otherwise improved outcomes in the overall patient population. There has been recent dis- cussion within the adult genitourinary surgical community focusing on the regionalization of radical prostatectomy to high volume centers, secondary to the belief this would provide patients with improved outcomes. Analysis compiled from the NIS concluded that low volume institutions experienced inferior outcomes relative to the highest volume centers, irrespective of approach for radical prostatectomy, supposedly demonstrating the importance of accounting for hospital volume when examining the benefit of a surgical technique (15). However, in the present study cohort when adjusted for hospital volume, there is a similar readmission rate among all institutions.

\subsection{Complex Chronic Conditions}

In 2011, Berry et al. used the PHIS database to characterize hospital readmissions between 2003 and 2008, finding $21.8 \%$ of patients experienced at least 1 readmission within 365 days of a prior admission; 2.9\% experienced 4 or more readmissions within 365 days, accounting for $18.8 \%$ of overall admissions (16). Of this $2.9 \%, 28.5 \%$ (2633) were re-hospitalized for a problem in the same organ system across all admissions during the interval. A retrospective analysis examined readmission among 1,083 patients hospitalized between 2006 and 2008 who were part of a structured, pediatric complex-care clinical program within 4 children's hospitals. Notably, almost half (47.1\%) of all complex-care clinical program patient admissions were related primarily to a surgical operation or major procedure (17). In our analysis, multivariate regression found males with CCCs were at increased risk of postoperative readmission; this is supported by previous data which has already identified the use of CCCs in models describing pediatric readmission rates $(18,19)$. Somewhat surprising was our finding that CCC was an independent predictor of readmission in males but not females. This could be secondary to a yet unidentified gap in the care of male patients. However, $83.5 \%$ of our cohort had a CCC, with $50 \%$ reporting a neuromuscular condition. As a result, the attempted use of CCCs for determining readmission risk is not ideal but was an attempt to find some way of quantifying risk. We attempted to find other predictors of readmission including specific conditions such as obesity but only initial LOS $>7$ days during the initial surgery yielded a 2-fold increase in likelihood of readmission. Rice-Townsend et al. utilized a retrospective analysis of the PHIS database to identify disease severity among patients as the primary factor associated with readmission after treatment of appendicitis (20). This factor was highly variable across hospital populations and significantly altered ranking of performance-based outliers. One of the limitations of our study is the inability to account for "disease severity", going back to the lack of unified, descriptive data that is needed to better stratify and describe the cohort of pediatric patients undergoing bladder reconstruction. Additional limitations include a lack of readmission data in situations where pediatric 
Cohen Set al.

patients were re-hospitalized in non-PHIS hospitals or patients were readmitted to a different PHIS hospital. This could be underestimating readmission frequency. On the other hand, patients may have been readmitted for other reasons outside of the initial bladder reconstruction, thus overestimating our readmission rates. The lack of sufficient finite data to characterize the reasons for readmission is a significant drawback in administrative databases, but we believe that a definite trend is seen in this study. The ability to generalize our results might also be limited to freestanding children's hospitals and does not represent the national norm, although it is estimated that $25 \%$ of children's hospitalizations occur within children's hospitals (National Statistics on Children, Healthcare Cost and Utilization Project, Agency for Healthcare Research and Quality, http://www.hcupnet.ahrq.gov/ (accessed March 30, 2013). Children treated at children's hospitals have a higher prevalence of CCCs than children at other hospitals, and this could potentially affect our results (21). In addition, our use of ICD-9 coding to identify patients for inclusion is always subject to the quality of initial data collection and may unintentionally lead to inclusion of inappropriate or exclusion of appropriate patients. With health care reform initiatives looming, the future of medicine will likely include bundled payments for inpatient surgical and outpatient post-hospitalization care, ensuring accountability for patient outcomes and inpatient utilization among care providers (22-24). Optimizing outcome by reducing morbidity is imperative. Despite advances in health care, pediatric bladder reconstruction is a significant endeavor with an essential likelihood for future admission at substantial cost. As evidenced in this study, readmission rates after pediatric bladder reconstruction have not improved over time. Nearly all centers have a similar readmission rate despite volume adjustment. Patient demographics and initial LOS may have a bearing on readmission risk. Collaborative quality improvement initiatives are required for this patient cohort.

\section{Acknowledgements}

We thank Eileen $\mathrm{H}$ Silva for editorial assistance.

\section{Authors' Contributions}

Study concept and design: Cohen and Chiang. Acquisition of data: Palazzi. Analysis and interpretation of data: Cohen, Palazzi, and Chiang. Drafting of the manuscript: Cohen and Chiang. Critical revision of the manuscript for important intellectual content: Cohen, Chiang, Marietti, and Kaplan. Statistical analysis: Palazzi and Chiang. Administrative, technical, and material support: Palazzi and Chiang. Study supervision: Chiang and Kaplan.

\section{References}

1. Biers SM, Venn SN, Greenwell TJ. The past, present and future of augmentation cystoplasty. BJU Int. 2012;109(9):1280-93.

2. Wiener JS, Antonelli J, Shea AM, Curtis LH, Schulman KA, Krupski TL, et al. Bladder augmentation versus urinary diversion in pa- tients with spina bifida in the United States.JUrol. 2011;186(1):161-5. 3. Lendvay TS, Cowan CA, Mitchell MM, Joyner BD, Grady RW. Augmentation cystoplasty rates at children's hospitals in the United States: a pediatric health information system database study. $J$ Urol. 2006;176(4 Pt 2):1716-20.

4. Schlomer BJ, Saperston K, Baskin L. National trends in augmentation cystoplasty in the 2000 s and factors associated with patient outcomes. J Urol. 2013;190(4):1352-7.

5. Obermayr F, Szavay P, Schaefer J, Fuchs J. Outcome of augmentation cystoplasty and bladder substitution in a pediatric age group. Eur J Pediatr Surg. 2011;21(2):116-9.

6. Metcalfe PD, Rink RC. Bladder augmentation: complications in the pediatric population. Curr Urol Rep. 2007;8(2):152-6.

7. Stein R, Schroder A, Thuroff JW. Bladder augmentation and urinary diversion in patients with neurogenic bladder: surgical considerations. J Pediatr Urol. 2012;8(2):153-61.

8. Scales CJ, Wiener JS. Evaluating outcomes of enterocystoplasty in patients with spina bifida: a review of the literature. J Urol. 2008;180(6):2323-9.

9. Feudtner C, Hays RM, Haynes G, Geyer JR, Neff JM, Koepsell TD. Deaths attributed to pediatric complex chronic conditions: national trends and implications for supportive care services. Pediatrics. 2001;107(6):E99.

10. McAdams-Demarco MA, Grams ME, Hall EC, Coresh J, Segev DL. Early hospital readmission after kidney transplantation: patient and center-level associations. Am J Transplant. 2012;12(12):3283-8.

11. Shankar N, Marotta P, Wall W, Albasheer M, Hernandez-Alejandro $\mathrm{R}$, Chandok N. Defining readmission risk factors for liver transplantation recipients. Gastroenterol Hepatol (NY). 2011;7(9):585-90.

12. Gore JL, Lai J, Gilbert SM, Urologic Diseases in America P. Readmissions in the postoperative period following urinary diversion. World J Urol. 2011;29(1):79-84.

13. Welk B, Herschorn S, Law C, Nam R. Population based assessment of enterocystoplasty complications in adults. $J$ Urol. 2012;188(2):464-9.

14. DeFoor WR, Heshmat S, Minevich E, Reddy P, Koyle M, Sheldon C. Long-term outcomes of the neobladder in pediatric continent urinary reconstruction. J Urol. 2009;181(6):2689-93.

15. Sammon JD, Karakiewicz PI, Sun M, Sukumar S, Ravi P, Ghani KR, et al. Robot-assisted versus open radical prostatectomy: the differential effect of regionalization, procedure volume and operative approach. JUrol. 2013;189(4):1289-94.

16. Berry JG, Hall DE, Kuo DZ, Cohen E, Agrawal R, Feudtner C, et al. Hospital utilization and characteristics of patients experiencing recurrent readmissions within children's hospitals. JAMA. 2011;305(7):682-90.

17. Berry JG, Agrawal R, Kuo DZ, Cohen E, Risko W, Hall M, et al. Characteristics of hospitalizations for patients who use a structured clinical care program for children with medical complexity. JPediatr. 2011;159(2):284-90.

18. Feudtner C, Levin JE, Srivastava R, Goodman DM, Slonim AD, Sharma $\mathrm{V}$, et al. How well can hospital readmission be predicted in a cohort of hospitalized children? A retrospective, multicenter study. Pediatrics. 2009;123(1):286-93.

19. Cohen E, Berry JG, Camacho X, Anderson G, Wodchis W, Guttmann A. Patterns and costs of health care use of children with medical complexity. Pediatrics. 2012;130(6):e1463-70.

20. Rice-Townsend S, Hall M, Barnes JN, Lipsitz S, Rangel SJ. Variation in risk-adjusted hospital readmission after treatment of appendicitis at 38 children's hospitals: an opportunity for collaborative quality improvement. Ann Surg. 2013;257(4):758-65.

21. Simon TD, Berry J, Feudtner C, Stone BL, Sheng X, Bratton SL, et al. Children with complex chronic conditions in inpatient hospital settings in the United States. Pediatrics. 2010;126(4):647-55.

22. Berry SA, Doll MC, McKinley KE, Casale AS, Bothe AJ. ProvenCare: quality improvement model for designing highly reliable care in cardiac surgery. Qual Saf Health Care. 2009;18(5):360-8.

23. Guterman S, Davis K, Stremikis K, Drake H. Innovation in Medicare and Medicaid will be central to health reform's success. Health Aff (Millwood). 2010;29(6):1188-93.

24. Moore KD, Coddington DC. Accountable care the journey begins. Healthc Financ Manage. 2010;64(8):57-63. 